\title{
Modulation of Microsaccades in Monkey during a Covert Visual Attention Task
}

\author{
Ziad M. Hafed, ${ }^{1}$ Lee P. Lovejoy, ${ }^{2}$ and Richard J. Krauzlis ${ }^{2,3}$ \\ ${ }^{1}$ Werner Reichardt Centre for Integrative Neuroscience, 72076 Tuebingen, Germany, ${ }^{2}$ Systems Neurobiology Laboratory, Salk Institute for Biological \\ Studies, La Jolla, California 92037, and ${ }^{3}$ Laboratory of Sensorimotor Research, National Eye Institute, Bethesda, Maryland 20892
}

The use of awake, fixating monkeys in neuroscience has allowed significant advances in understanding numerous brain functions. However, fixation is an active process, with the occurrence of incessant eye movements, including rapid ones called microsaccades. Even though microsaccades have been shown to be modulated by stimulus and cognitive processes in humans, it is not known to what extent these results are similar in monkeys or why they occur. Here, we analyzed the stimulus-, context-, and attention-related changes in microsaccades while monkeys performed a challenging visual attention task. The distributions of microsaccade times were highly stereotypical across thousands of trials in the task. Moreover, in epochs of the task in which animals anticipated the occurrence of brief stimulus probes, microsaccade frequency decreased to a rate of less than one movement per second even on long multisecond trials. These effects were explained by the observation that microsaccades occurring at the times of the brief probes were sometimes associated with reduced perceptual performance. Microsaccade directions also exhibited temporal modulations related to the attentional demands of the task, like earlier studies in humans, and were more likely to be directed toward an attended location on successfully performed trials than on unsuccessfully completed ones. Our results show that microsaccades in nonhuman primates are correlated with the allocation of stimulus-evoked and sustained covert attention. We hypothesize that involvement of the superior colliculus in microsaccade generation and attentional allocation contributes to these observations. More importantly, our results clarify the potential role of these eye movements in modifying behavior and neural activity.

\section{Introduction}

Vision studies often enforce fixation to minimize interpretational problems caused by eye movements. However, during fixation, the eyes never remain still, but instead exhibit continuous slow drifts in position interspersed with rapid saccadic displacements (Ratliff and Riggs, 1950; Barlow, 1952; Ditchburn and Ginsborg, 1953; Zuber and Stark, 1965; Martinez-Conde et al., 2004). These "microsaccades" have properties similar to larger saccades at behavioral (Zuber and Stark, 1965) and neurophysiological (Van Gisbergen et al., 1981; Hafed et al., 2009; Hafed, 2011) levels, suggesting that they may pose similar interpretational ambiguities. For example, microsaccades can cause visual suppression (Zuber and Stark, 1966; Beeler, 1967; Hafed and Krauzlis, 2010), like the known saccadic suppression phenomena (Diamond et al., 2000; Ross et al., 2001). Thus, understanding how microsaccades are modulated during visual experiments is not only important for understanding how these movements are generated,

Received June 18, 2011; revised Aug. 15, 2011; accepted Aug. 19, 2011.

Author contributions: Z.M.H., L.P.L., and R.J.K. designed research; Z.M.H., L.P.L., and R.J.K. performed research; Z.M.H., L.P.L., and R.J.K. analyzed data; Z.M.H., L.P.L., and R.J.K. wrote the paper.

This work was supported by the Werner Reichardt Centre for Integrative Neuroscience (Z.M.H.), the Institute for Neural Computation and the Aginsky Scholars Award Program (L.P.L.), and National Institutes of Health Grant EY12212 (R.J.K.)

Correspondence should be addressed to Ziad M. Hafed, Werner Reichardt Centre for Integrative Neuroscience, Paul Ehrlich Strasse 17, 72076 Tuebingen, Germany. E-mail: ziad.m.hafed@cin.uni-tuebingen.de.

DOI:10.1523/JNEUROSCI.3106-11.2011

Copyright $\odot 2011$ the authors $\quad 0270-6474 / 11 / 3115219-12 \$ 15.00 / 0$ but it is also necessary for properly interpreting experiments requiring prolonged fixation.

In humans, microsaccades have been found to exhibit consistent event-related modulations during tasks involving covert attention (Hafed and Clark, 2002; Engbert and Kliegl, 2003; Laubrock et al., 2005), but several unanswered questions remain concerning these results. First, these results are not universally accepted (Tse et al., 2002; Horowitz et al., 2007) [but see Laubrock et al. (2010) for a possible reconciliation]. Also, it is not clear how these results extend to fixation sustained over many seconds, or when attention is allocated without a sensory cue. Finally, it is not entirely clear why these modulations occur in the first place. Clarifying these issues is a necessary step toward understanding the mechanistic interactions between microsaccades and perceptual/cognitive processes.

In the monkey, there is both controversy and a rarity of investigations concerning the relationship between microsaccades, task-related events, and neural activity. Save one study exploring exogenous capture of attention (Brien et al., 2009), and some references to microsaccadic modulations that may relate to attention (Deaner and Platt, 2003; Corneil et al., 2008; Cui et al., 2009), there has been no systematic attempt to analyze microsaccades in monkeys performing cognitive and perceptual tasks. Moreover, microsaccades have recently garnered attention because of how these movements may affect behavioral and neural data (Bosman et al., 2009; Herrington et al., 2009; Hafed and Krauzlis, 2010), but these studies did not examine how the patterns of microsaccades themselves are influenced by the tasks. Finally, the effects of 
microsaccades on perception remain hotly debated. For example, it is still contentious whether microsaccades cause enhancement, suppression, or both of V1 neural activity (Leopold and Logothetis, 1998; Martinez-Conde et al., 2000; Kagan et al., 2008; Dimigen et al., 2009).

Here, we analyzed thousands of trials in monkeys performing a demanding visual attention task (Lovejoy and Krauzlis, 2010). We found very orderly effects supporting the idea that microsaccades are caused by neural activity representing behaviorally relevant spatial locations. We also found that microsaccades can be associated with reduced perceptual performance under some circumstances. Finally, we observed task-related inhibition of microsaccades, suggesting that monkeys may have some degree of voluntary control over these movements.

\section{Materials and Methods}

Our laboratory setup was identical to that described recently (Hafed and Krauzlis, 2008; Hafed et al., 2008, 2009; Lovejoy and Krauzlis, 2010).

\section{Animal preparation}

We collected behavioral data from two ( $\mathrm{J}$ and $\mathrm{M}$ ) adult, male rhesus monkeys (Macaca mulatta) that were 10-15 years of age and weighed 12-15 kg. The monkeys were prepared using standard surgical techniques that have been described in detail previously (Krauzlis, 2003), and all experimental protocols for the monkeys were approved by the Institutional Animal Care and Use Committee and complied with United States Public Health Service policy on the humane care and use of laboratory animals.

\section{Behavioral tasks}

Monkeys performed the selective attention tasks described by Lovejoy and Krauzlis (2010) (also see Fig. 1). Briefly, monkeys initially fixated a central dot like that described by Hafed et al. (2009). During fixation, four stochastic motion patches $\left(8.5^{\circ}\right.$ diameter, centered at $8.2^{\circ}$ eccentricity) were presented, one in each quadrant of the visual field. At $480 \mathrm{~ms}$ before patch onset, one of the four patches was cued with a colored ring surrounding the patch location. The cue disappeared $480 \mathrm{~ms}$ after patch onset, and after an additional variable delay (480-4000 ms, geometrically distributed), a brief ( $160 \mathrm{~ms}$ ) pulse of coherent motion appeared in the cued patch. A second motion pulse with different direction appeared at the same time in the diametrically opposite location, defined as the "foil location." The directions of the motion pulses were selected from four possible diagonal directions: up to the right, down to the right, down to the left, and up to the left. The monkeys were rewarded for fixating the central dot for the entire duration of the trial and then correctly reporting the direction of the cued motion pulse, regardless of the location of the cue, and regardless of the motion direction at the foil location.

Monkeys reported the motion direction at the cued location in two different ways, in separate versions of the task. In the "saccade" version of the task, the monkeys reported their perceived direction with a saccade in the same direction. For example, if the motion at the cued location was directed down to the right (regardless of cue location), the response saccade was directed to the lower right quadrant. Four choice dots appeared after the motion pulse ended to assist the monkeys in guiding their response saccades (see Fig. 1). In the "button press" version of the task, the stimuli and instructions were identical, except that the choice dot frame that appeared at the end of the motion pulse (see Fig. 1) was replaced with another frame. This alternative choice dot frame consisted of the same fixation spot, but now surrounded by a square of the size of the fixation window $\left( \pm 1^{\circ}\right.$ around the fixation spot) whose four corners were highlighted (i.e., we presented a white square with small gaps in the middle of each side to only highlight the corners). The monkeys' task was to maintain fixation within this window after motion pulse end and to report their perceived direction by pressing one of four buttons arranged to represent the four quadrants corresponding to the four possible motion directions. The buttons were not within the monkeys' view.

We collected 21,621 trials in 36 sessions from the saccade variant of the selective attention task, and 24,813 trials in 44 sessions from the button press variant. Cue locations were typically blocked for 40 trials at a time, and we collected data uniformly across all possible cue locations.

\section{Data analysis}

Eye movement detection and classification. Eye movements were sampled at $1 \mathrm{kHz}$. Saccades and microsaccades were detected using velocity and acceleration thresholds as described previously (Krauzlis and Miles, 1996; Hafed et al., 2009). Specifically, our algorithm identified the point of peak radial eye velocity (above a threshold parameter, which we initially set to $8 \%$ ) and flagged it as part of a saccade. Then, flanking regions around this point during which eye velocity remained higher than the velocity threshold were included as part of the same saccade. After that stage, the start and end points of the saccade being flagged were refined by finding the time points at which eye acceleration in the direction of the saccade exceeded (for saccade start) or went below (for saccade end) a second threshold parameter (the acceleration threshold, which we initially set to $550 \% \mathrm{~s}^{2}$ ). Our choice of velocity and acceleration thresholds was made empirically to avoid erroneous flagging of drifts/noise while at the same time accounting for the fact that microsaccades are generally slower than larger voluntary saccades. Even with this choice, there may have been cases in which the algorithm failed. Thus, after running the saccade detection algorithm, we visually inspected every trial and each individual microsaccade, and we manually verified that the algorithm did not erroneously miss a microsaccade or falsely detect one. After all of these steps, we confirmed the accuracy of our detection algorithm and manual inspection by plotting the main sequence curve for microsaccades and comparing it with that of larger saccades (see, for example, Fig. $2 B$ ).

We detected and analyzed all microsaccades occurring before the monkeys' behavioral response (either saccade or button press). We excluded from the analysis all trials with movements larger than $1^{\circ}$ during the required fixation intervals. These were few, and the movements that we analyzed were typically significantly smaller than $1^{\circ}$ (mean, 16 min arc; also see Fig. 2 for sample trials and sessions).

We classified microsaccade directions according to the four quadrants of the visual display. For example, if the cue was in the upper right quadrant, microsaccades were classified as being in the direction of the cued quadrant if their direction was up and to the right. We also classified microsaccade directions according to the physical quadrant toward which the movements were directed; quadrants were numbered sequentially in a clockwise manner (i.e., quadrant 1 was defined as the upper right quadrant, quadrant 2 the lower right, quadrant 3 the lower left, and quadrant 4 the upper left).

Time course of microsaccade frequency and direction. To study how stimulus, attentional, and context-related factors could influence microsaccades, we plotted the onset times of these movements as rasters of dots in which each row corresponded to an individual trial and each location of a dot along the $x$-axis corresponded to the onset time of a movement. We either aligned such plots on trial onset, to study early stimulusinduced changes in microsaccades, or on trial end (motion pulse onset), to study late interactions with response selection and execution. We also sorted trials by factors such as trial length and reaction time (RT). Summary histograms of the rasters were also obtained to estimate how microsaccade rate changed in different trial epochs. To obtain such a rate, we used a running temporal bin of width $80 \mathrm{~ms}$. In each such bin, we estimated the instantaneous rate, and we successively moved the bin center in $5 \mathrm{~ms}$ steps. Because trials were of variable length, estimates of microsaccade rate at very long intervals after trial onset were made from fewer trials than estimates of rate early in the trials.

To study the influence of attended locations on microsaccade directions, we repeated the time course analyses above but on the "differential fraction of microsaccades" that were directed toward a given quadrant. For each quadrant, we first obtained the frequency of movements directed toward that quadrant as a function of time, regardless of cue location. We then measured the same frequency of movements but when the cue was either in the same quadrant, the opposite quadrant (meaning that the foil was in the same quadrant), or neither. The differential fraction curve was plotted as the difference between the two curves (with positive indicating a bias toward the quadrant and negative indicating a 


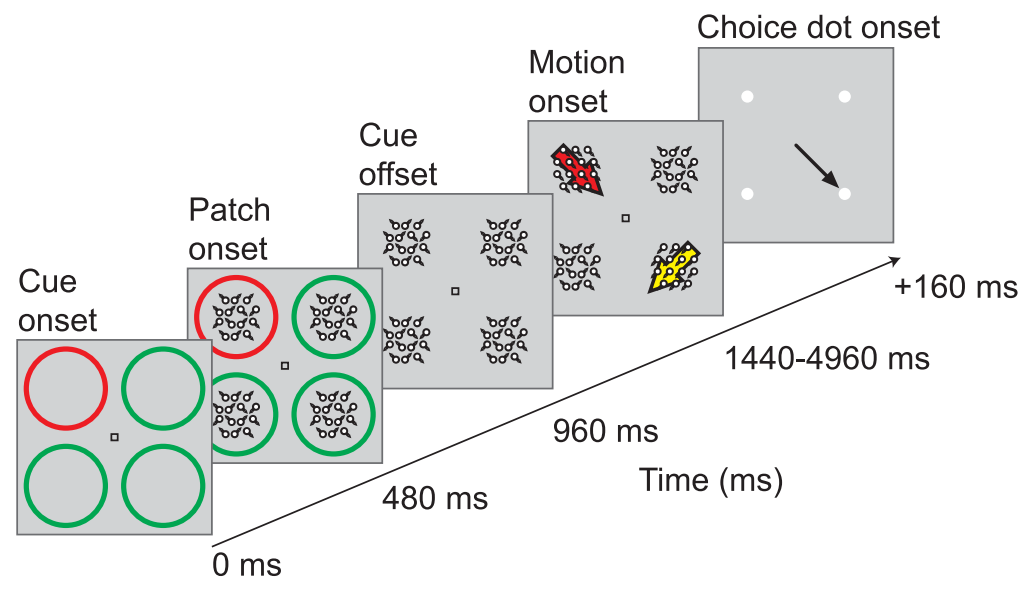

Figure 1. Sustained covert visual attention task. Monkeys fixated a small fixation spot at the center of the display. At trial onset, four peripheral rings appeared, with the odd-colored ring (red in the figure) cueing the monkeys to selectively attend to stimuli within the same quadrant. After $480 \mathrm{~ms}$, four patches of random dot motion stimuli ( $0 \%$ coherence) appeared within each ring. The cue rings disappeared after another $480 \mathrm{~ms}$, signaling to the monkeys the impending occurrence of a brief coherent motion pulse in both the cued location and the diametrically opposite quadrant, defined as the foil. The motion pulses (indicated schematically with red and yellow arrows in the figure) lasted for $160 \mathrm{~ms}$ and they could occur at any time between $\sim 480$ and $\sim 4000 \mathrm{~ms}$ after cue offset. After the pulses, four choice dots appeared, and the monkey was instructed to look at the dot corresponding to the direction of motion at the cued location (down to the right in the shown example). In the button press version of the task, the choice dots were replaced by a square around the fixation spot (see Materials and Methods), and monkeys had to remain fixating within this square and press a button to indicate their perceptual judgment.

bias away from it). The $95 \%$ confidence intervals for these directional evolution curves were estimated across all quadrants and all cue locations using a bootstrap of the entire array of detected microsaccades (1000 iterations, with replacement) (Efron and Tibshirani, 1993). We next estimated the timing of cue-evoked reversals in microsaccade directions by measuring the maximum of the differential fraction curves for microsaccades directed toward either the cued or foil locations (in the first 1000 $\mathrm{ms}$ after cue onset). This approach of obtaining a differential fraction of microsaccades directed toward a given quadrant (cued, foil, or neither) allowed us to isolate the directional modulations of microsaccades by stimulus, attentional, and context-related factors from inherent biases in direction that were idiosyncratically present in each monkey. For example, in monkey J, there tended to be a strong bias for microsaccades into quadrant 2 even without cueing (for example, see Fig. $2 \mathrm{~A}$, left), and our goal was to identify how cueing or attention or behavioral context modulated directions on top of this basic bias. For other analyses of microsaccade directions, we also plotted the absolute frequency of microsaccades that were directed toward a given quadrant (cued, foil, or neither) within a given interval (i.e., not a differential fraction), and similar conclusions were reached (for example, see Figs. 6, 7).

Relationship between microsaccades and behavioral performance. We measured both RT and the fraction of correctly performed trials (or the fraction of choices guided by the foil-defined as "fraction of foil choices") as a function of when a microsaccade happened relative to motion pulse onset. To obtain a time course of these effects, we performed the analyses by binning the closest microsaccade to motion pulse onset according to its time of occurrence. Specifically, and just as we did for microsaccade rate and directional evolutions, we used a running average with temporal bins $(60 \mathrm{~ms}$ width for RT and $120 \mathrm{~ms}$ width for fraction of correct or foil choices) successively moved in $5 \mathrm{~ms}$ steps relative to the time of motion pulse onset. In addition, we repeated the same analyses but after separating microsaccades based on whether they were directed toward the cued or foil location. For comparison to microsaccade-free situations, we measured baseline RT and fraction of correct or foil choices on all trials during which no microsaccades occurred within $250 \mathrm{~ms}$ from motion pulse onset. When estimating the time at which RT or the fraction of correct/foil choices was altered around the time of microsaccades (i.e., the time at which these two performance measures deviated from the no-microsaccade baseline), we used the time at which the $95 \%$ confidence intervals of RT or fraction of correct/foil choices and the corresponding baseline measurements ceased to overlap.

\section{Results}

We trained monkeys to perform a demanding covert attention task (Fig. 1) (Lovejoy and Krauzlis, 2010). In this task, monkeys were cued to monitor one quadrant of the visual display and discriminate the direction of motion in a brief (160ms-long) motion pulse that could occur after up to several seconds of maintained fixation. The monkeys' selective attention to the cued quadrant was further challenged by the simultaneous presentation of a distracting "foil" motion pulse of equal strength at the diametrically opposite location. As described in more detail by Lovejoy and Krauzlis (2010), monkeys performed the motion discrimination task diligently: they achieved $\sim 64 \%$ success rate, and importantly, the majority ( $\sim 55 \%$ ) of their errors involved reporting the direction of the foil motion, rather than random guesses or lapses in following the instructions to report motion direction. Thus, the cued and foil locations appeared to be particularly important for the monkeys' performance in this task (Lovejoy and Krauzlis, 2010).

Analysis of the monkeys' eye movements during task performance revealed that microsaccades occurred during the majority of trials. Figure $2 \mathrm{~A}$ shows sample eye position traces of one monkey $(\mathrm{J})$ from individual trials in the saccade (left) and button press (right) variants of the covert attention task. In each of these trials, microsaccades are marked using green symbols. In addition, for the saccade variant of the task, the response saccade of $\sim 8^{\circ}$ amplitude to the lower left quadrant is also shown (marked in yellow). As can be seen, several microsaccades occurred during each trial. In addition, the button press trial showed a (slightly larger) movement occurring very close to the button press response. In each of these sample sessions, 1320 microsaccades occurred in 352 trials of the saccade session, and 1119 microsaccades occurred in 176 trials of the button press session. These movements are summarized in Figure $2 B$, which also shows that the movements in these two sample sessions conformed to the main sequence of saccades and microsaccades (Zuber and Stark, 1965). Moreover, all trials in each of these sample sessions contained microsaccades.

In the following, we describe modulations in the frequency and directions of microsaccades during the covert attention task, as well as the different potential influences these small eye movements may have had on behavioral performance. We first present results for the saccade version of the task, and then describe how the microsaccade-related phenomena changed in the button press version.

\section{Task events caused stereotypical changes in the frequency of microsaccades}

Across thousands of trials in the saccade task, the temporal pattern of microsaccade onsets in relation to cue onset, cue offset, and the appearance of the $0 \%$ motion patches was remarkably consistent. Figure $3 A$ shows a "raster plot" of microsaccade onset times across all trials for each monkey, as well as a summary 
histogram across trials, showing the evolution of microsaccade frequency (or rate) as a function of time. The trials in the raster plot were sorted by trial length (defined as the time between cue onset and motion pulse onset). In addition, the onset times of the monkeys' response saccades are plotted as green dots in the rasters. Stimulus events like cue onset/offset and $0 \%$ motion patch onset induced the generation of microsaccades after some delay, with idiosyncratic differences between the monkeys. Specifically, before trial onset, microsaccades had a baseline rate or likelihood of occurrence of $\sim 1.2$ movements per second in monkey $\mathrm{M}$ and $\sim 2.1$ movements per second in monkey J. Approximately 200-250 ms after cue onset (Fig. $3 A$, leftmost arrow in the rate curves), a peak in microsaccade rate was induced in each monkey, but the increase was significantly larger (and later) in monkey $\mathrm{J}$ than in monkey $\mathrm{M}$ (peak rate occurred at $t=245 \mathrm{~ms}$ for monkey J and $190 \mathrm{~ms}$ for monkey M). Approximately 200-250 ms after patch onset (Fig. 3A, middle arrow in the rate curves), another set of microsaccades was induced (with similar latencies relative to patch onset; $250 \mathrm{~ms}$ for $\mathrm{J}$ and $180 \mathrm{~ms}$ for $\mathrm{M}$ ), and this effect was much stronger than the cue onset effect in monkey M. Finally, cue offset had a similar effect in both monkeys, although the peak in microsaccade rate that was induced by this stimulus event was weaker than that for cue and $0 \%$ motion patch onset (Fig. 3A, rightmost arrow in the rate curves). These stimulus-induced changes in microsaccade rate are similar to ones observed previously in humans (Hafed and Clark, 2002; Engbert and Kliegl, 2003; Laubrock et al., 2005).

Starting from approximately the earliest possible onset time of the brief motion pulse in the task (Fig. $3 A$, dashed vertical black line), both monkeys exhibited a strong reduction in microsaccade frequency relative to earlier epochs in the trials. This occurred even though the monkeys fixated for prolonged periods of times, and even though trial length was geometrically distributed to minimize potential effects of temporal anticipation (Lovejoy and Krauzlis, 2010). For example, Figure $3 A$ shows that, after $\sim 1440$ ms from cue onset (dashed vertical black line in the figure), microsaccade rate in both monkeys dropped to below one movement per second (but was never completely abolished), which was well below the baseline rate before trial onset (dashed horizontal gray line), and certainly much lower than the induced microsaccade rates after stimulus events. In addition, when we aligned the data on motion pulse onset (Fig. $3 B$ ), we found that microsaccade rate was consistently lower than one movement per second at motion pulse onset (even though trial length was variable), and it decreased further to 0 as the monkeys prepared their $\sim 8^{\circ}$ response saccades after motion pulse end. Thus, anticipation of the brief motion pulses at the cued and foil locations appeared to be associated with a significant reduction in microsaccade frequency, even though the monkeys maintained fixation over long
Saccade Task

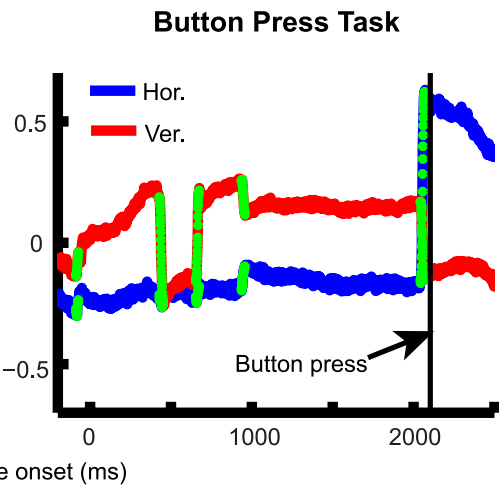

Time from cue onset $(\mathrm{ms})$
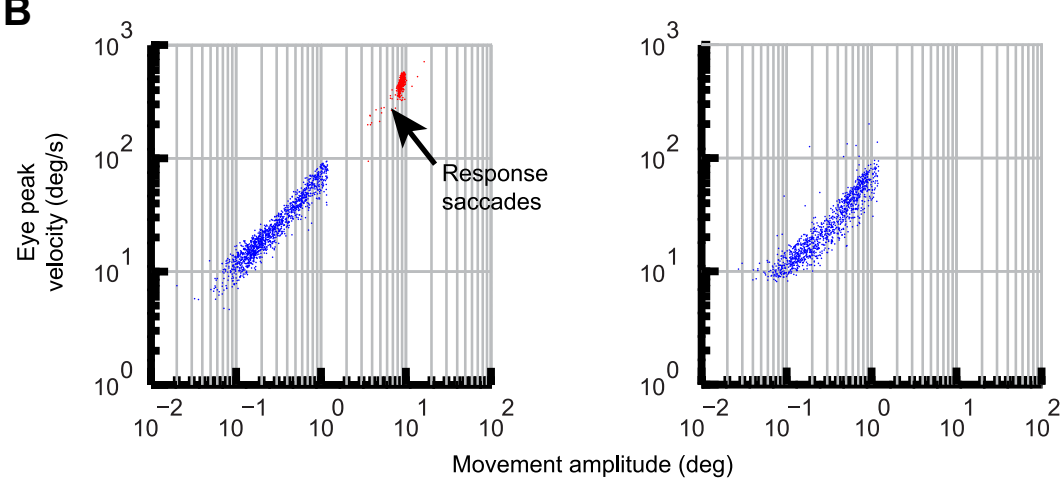

Movement amplitude (deg)

Figure 2. Sample fixational eye position data from the task. $\boldsymbol{A}$, Eye position traces from one representative trial from each of the fhe trial and before the response saccade. The right panel shows similar data from a trial in the button pres (1) button press. $\boldsymbol{B}$, Plots of saccade peak velocity versus amplitude ("main sequence") for all trials from the same sessions of the sample trials in $A$ indicating the range of eye movements observed. In the left panel, the red dots indicate the response saccades at the ends of trials in this task variant. As can be seen, the range of microsaccade amplitudes in the saccade and button press task variants was similar (compare blue dots in the right and left panels).

intervals. Our analyses of the relationship between microsaccades and behavioral performance, described below, provide a possible reason for this inhibition.

\section{Microsaccades at the time of motion pulse onset were} associated with changes in perceptual performance

Sorting the trials of the saccade task based on reaction time in Figure $3 B$ revealed an additional feature of the data: a negative correlation between microsaccade onset time and the monkeys' RTs. Specifically, inspection of the figure reveals that short RT trials in both monkeys (Fig. $3 B$, bottom set of trials in the rasters) were associated with a lower probability of microsaccades occurring around motion pulse onset (i.e., there was a lower density of dots in the rasters). To explore this further, we analyzed the relationship between the microsaccade closest to motion pulse onset and the monkeys' RT, and also the frequency of correct responses.

Microsaccades occurring around the motion pulse interval were associated with longer reaction times. In an analysis similar to that performed in microsaccadic suppression studies (Zuber and Stark, 1966; Beeler, 1967; Hafed and Krauzlis, 2010), we plotted the monkeys' RT as a function of when a microsaccade occurred relative to motion pulse onset (Fig. $4 A$ ). We then compared these reaction times with those observed on the trials in which there were no microsaccades occurring within $250 \mathrm{~ms}$ of motion pulse onset (Fig. $4 A$, red line baselines). Both monkeys 

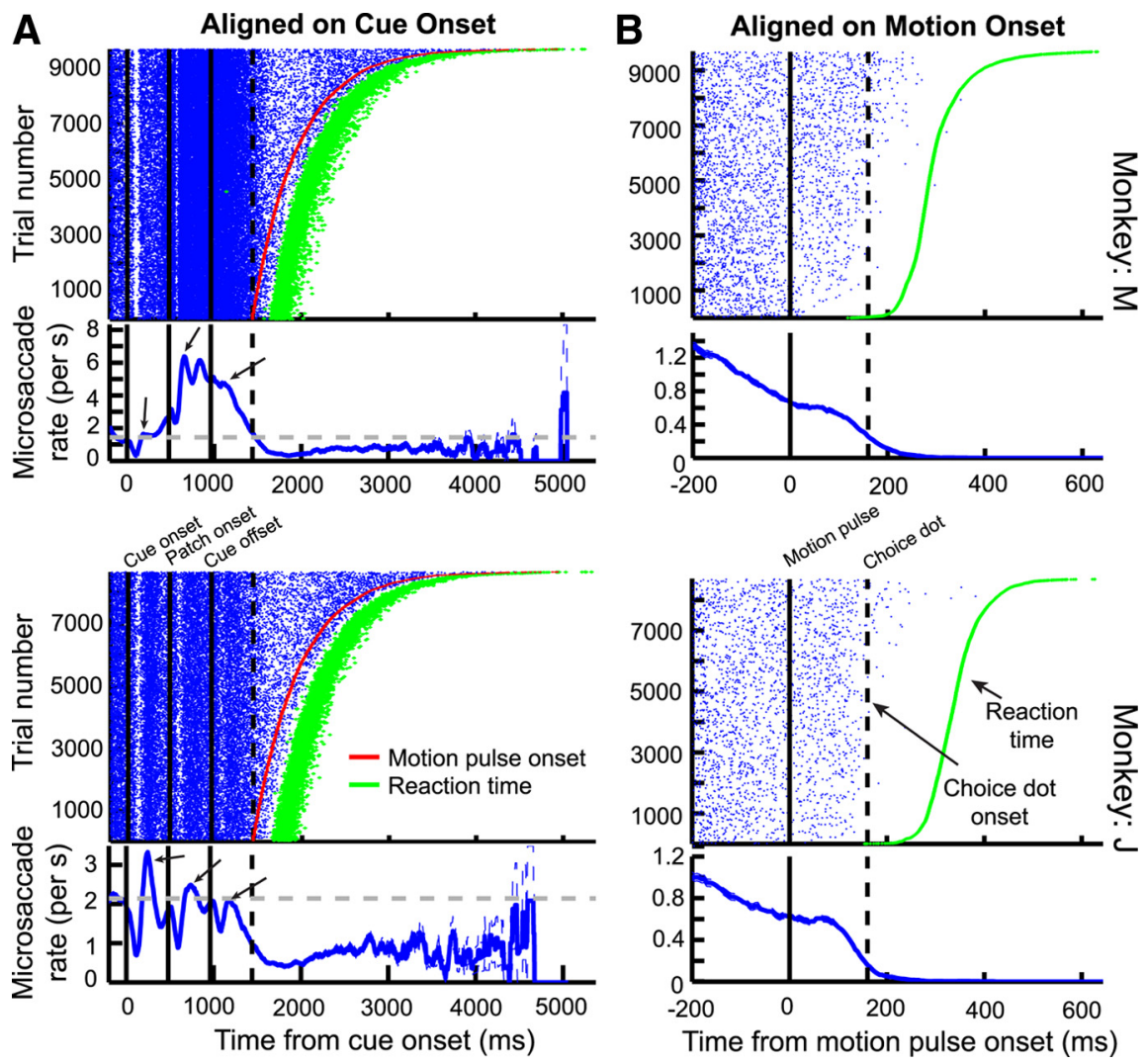

Figure 3. Stimulus cue events systematically induced microsaccades; anticipation of the motion pulses at trial end systematically suppressed microsaccades. $\boldsymbol{A}$, For each monkey, the top panel shows microsaccade onset times in all trials, when the trials were sorted by length. Each dot represents a microsaccade onset time, and the row on which the dot is drawn indicates the sorted trial number. Red indicates motion pulse onset, and green indicates the response saccade occurrence. Below this graph, a summary histogram is shown in the form of microsaccade rate per second. The three black vertical lines indicate cue onset, patch onset, and cue offset, respectively. The dashed black vertical line indicates when the earliest motion pulse could occur. In both monkeys, cue and stimulus events caused volleys of microsaccades shortly later (and above the pretrial baseline rate indicated by the gray dashed line). In addition, anticipation of motion pulse onset (through knowledge of the earliest possible pulse time) caused a dramatic reduction of microsaccade rate below baseline. $\boldsymbol{B}$, A subset of the data in $\boldsymbol{A}$ but close to motion pulse onset. The trials in the top panel for each monkey are now sorted by RT (indicated by the green dots). Microsaccades ceased completely before the response saccades, as expected. Note how short RT trials were associated with fewer microsaccades around pulse and choice dot onset in both monkeys. Error bars (when visible) indicate SEM.

exhibited elevated reaction times when microsaccades occurred around pulse onset, even for microsaccades occurring before $(\sim 8$ $\mathrm{ms}$ in monkey $\mathrm{M}$ and $\sim 71 \mathrm{~ms}$ in monkey $\mathrm{J}$ ) motion pulse onset (Fig. 4A, compare blue and red curves). These data are reminiscent of microsaccadic suppression phenomena, which we recently found can significantly increase saccadic reaction times (Hafed and Krauzlis, 2010); other studies (Bosman et al., 2009; Herrington et al., 2009) have also found evidence of changes in performance in association with microsaccades.

The temporal pattern of reaction time elevation associated with microsaccades also revealed later interactions, toward the end of the motion pulse. In both monkeys, for microsaccades occurring shortly after pulse onset, there was a slight dip in RT toward the baseline value after the initial increase (Fig. 4A). However, for even later microsaccades, there was a second (larger) increase in RT, peaking for microsaccades that occurred $\sim 160$ ms after motion pulse onset (i.e., at the end of the motion pulse, Fig. $4 A$ ). This second increase in RT is likely to be another manifestation of microsaccadic suppression (Hafed and Krauzlis, 2010), but associated with the onset of the choice dot frame at trial end (see last frame of Fig. 1). Thus, RT increases indicate that microsaccades occurring at critical intervals in this task could be associated with a decrease in the speed of the monkeys' performance.

Microsaccades occurring near motion pulse onset were also associated with changes in the monkeys' success rates. We performed a similar analysis to that in Figure $4 A$, but for the fraction of correctly performed trials (Fig. $4 B$ ). In both monkeys, microsaccades occurring during the motion pulses were associated with a decrease in the monkeys' correct performance. The temporal pattern of results observed in this analysis was slightly different from that concerning RT data that we found in Figure $4 A$. Specifically, we did not see evidence of two dips in performance (like the two elevations in RT), probably because even if microsaccadic suppression were to take place for choice dot onset (and affect RT), this happened too late to influence perception of the motion pulse itself. However, microsaccades at the onset of the motion pulse, or during it, can influence the perception of the motion pulse (whether through microsaccadic suppression, or simply due to retinal image blurring by eye movements, or due to other unknown factors). Together, these results on reaction time (Fig. 4A) and fraction correct (Fig. $4 B$ ) show that, at least under some circumstances, microsaccade occurrence can be correlated with a decrease in perceptual performance. As we show later (see Figs. 7, 8), this effect, as well as additional changes in the choices of the monkeys with the foil motion stimulus, actually depends on the direction of microsaccades relative to the cued and foil locations (reflecting the successful or failed allocation of covert attention).

The above two sets of results (Fig. 4) therefore provide a possible explanation for the strong reduction in microsaccade rate in Figure 3 starting from the approximate minimum time of expected motion pulse onset until trial end. The monkeys' expectations of a brief motion pulse anywhere from $\sim 1440 \mathrm{~ms}$ after trial onset may have led to suppression of microsaccades (starting from the earliest possible pulse time) to avoid the potential detrimental effects that we observed in Figure 4 .

\section{Microsaccade directions reflected the behavioral relevance of spatial locations}

To help understand why microsaccades occurred at all in the task, we next analyzed the influence of stimulus events and behavioral relevance on the distribution of microsaccade directions. We found that early stimulus-induced microsaccades predominantly reflected the two quadrants containing future discrimination stimuli-the cued and foil stimulus locations-and were less likely to be directed toward the two other quadrants (referred to as "neither"). Figure 5 illustrates this point by plotting the differential fraction of microsaccades that were directed toward a given quadrant (see Materials and Methods) as a function of time from trial onset. In both monkeys, the early stimulus-induced micro- 


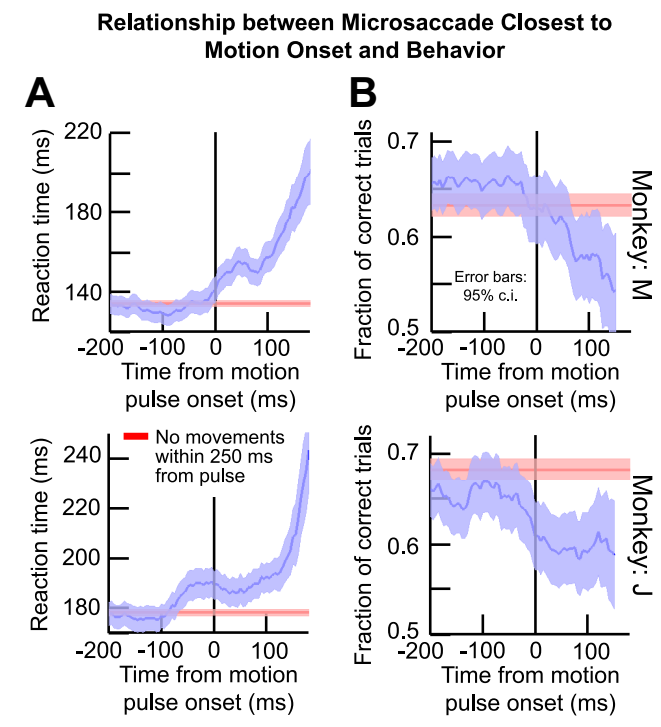

Figure 4. Monkey performance when microsaccades occurred around motion pulse onset. $\boldsymbol{A}, \mathrm{RT}$ as a function of when the microsaccade closest to motion pulse onset occurred in a trial. The red line shows baseline RT from all trials in which there were no microsaccades occurring within 250 ms from motion pulse onset. RTs were significantly longer than baseline when microsaccades occurred around motion pulse onset. Moreover, at a later interval (around 160 $\mathrm{ms}$ ), there was a further increase in RT relative to baseline. $\boldsymbol{B}$, Same as $\boldsymbol{A}$ but for the fraction of correct trials that the monkeys achieved. In both monkeys, a microsaccade occurring around and during the motion pulse was correlated with a reduction in the number of correct responses (see Figs. 7 and 8 for similar analyses in which microsaccade direction was taken into account, and in which we also measured the fraction of foil choices by the monkeys). Note that we could not map RT and fraction of correct trials for longer time intervals after motion pulse onset because of the scarcity of movements at the ends of trials (Fig. 3). Error bars in all panels indicate $95 \%$ confidence intervals.

saccades evident in Figure 3 were not randomly distributed in direction, but were correlated with the behaviorally relevant stimulus locations. Specifically, the earliest microsaccades after trial onset were directed back and forth between the cued and foil locations. In monkey $\mathrm{M}$, the first bias was toward the cued location (with a peak effect occurring $\sim 130 \mathrm{~ms}$ after trial onset; blue dashed line) before a reversal toward the foil (peaking at $\sim 350$ ms; blue dashed line). In monkey $\mathrm{J}$, the reversals in direction occurred significantly slower after cue onset than in monkey $\mathrm{M}$, and the first bias (peaking at $\sim 300 \mathrm{~ms}$ ) was toward the foil before reversing back toward the cue (with a peak effect at $\sim 640 \mathrm{~ms}$ ). Interestingly, monkey $\mathrm{M}$ generally exhibited significantly faster reaction times than monkey J ( 136 vs $180 \mathrm{~ms} ; p<0.0001, t$ test; also compare top and bottom panels of Fig. $4 A$ ), and this difference was also evident in the more rapid reversals in microsaccade direction. For both monkeys, movements with directional biases toward neither the cued nor the foil locations (black curves) were significantly decreased from trial onset and did not vary much over time. Thus, early cue-induced microsaccade directions were biased by the cued spatial location, as in human studies (Hafed and Clark, 2002; Engbert and Kliegl, 2003).

The directional biases toward either the cued or foil locations persisted for the later microsaccades that occurred during the sustained interval of attentional allocation, even though the overall rate of these movements was significantly reduced. These biases are evident in Figure 5: for microsaccade onset times toward the end of the plotted interval in the figure, the curves for movements toward the cued or foil locations were consistently $>0$, and the curves for movements toward the remaining two irrelevant locations were consistently $<0$. For example, for microsaccades

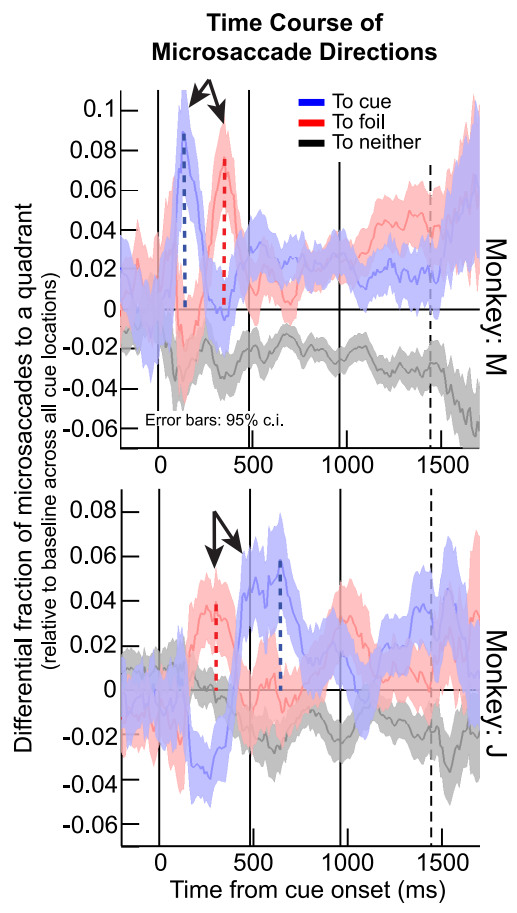

Figure 5. Microsaccade directions after cue onset reflected the possible locations of subsequent motion pulse stimuli needed to perform the task. For each monkey, we plotted the differential fraction of microsaccades that were directed toward a given quadrant when the quadrant was cued (blue), a foil (red), or completely irrelevant (black). Positive means a bias toward the indicated location. Early after cue onset, microsaccade directions in both monkeys preferentially switched between cued and foil locations and showed no particular bias toward the two irrelevant quadrants in the display. However, each monkey showed a different temporal sequence, with M showing the first population of microsaccades being biased toward the cue before reversing to the foil and J showing the first population of microsaccades being biased toward the foil before reversing to the cue. Later in the trials, and in anticipation of the earliest possible motion pulse onset (starting from the dashed vertical line), microsaccades were consistently directed toward either the cued or foil quadrant but not toward the two other irrelevant regions of the display. The three black vertical lines indicate cue onset, patch onset, and cue offset, respectively. The colored drop lines estimate the local peaks in the directional curves and demonstrate the different timing of directional effects across the two monkeys. Error bars indicate $95 \%$ confidence intervals.

occurring around $\sim 1500 \mathrm{~ms}$ after cue onset (i.e., around the earliest possible motion pulse occurrence), directional biases toward the cued and foil locations (blue and red curves) and directional biases away from the remaining two irrelevant locations (black curves) were consistently observed in the two monkeys.

The persistence of directional biases toward the cued and foil locations and away from the irrelevant ones suggests that around motion pulse onset there should again be a consistently higher likelihood of observing microsaccades that are directed toward either the cued or foil locations than toward the other two other locations. To test this explicitly, we analyzed the directions of all microsaccades occurring within $70 \mathrm{~ms}$ from motion pulse onset (Fig. 6). The majority of microsaccades occurring in this later interval, specifically chosen to be dissociated from earlier stimulus-induced effects on microsaccades, had directions congruent with the cued and foil locations and incongruent with the remaining two irrelevant locations. Thus, even though microsaccades were less frequent before the onset of the motion pulse (Figs. 3, 4), and even though these microsaccades occurred well after stimulus-induced changes in microsaccades had subsided (Fig. 3), they nonetheless reflected the behavioral relevance of specific spatial locations in the scene. 
Movements within $70 \mathrm{~ms}$ of Motion Onset
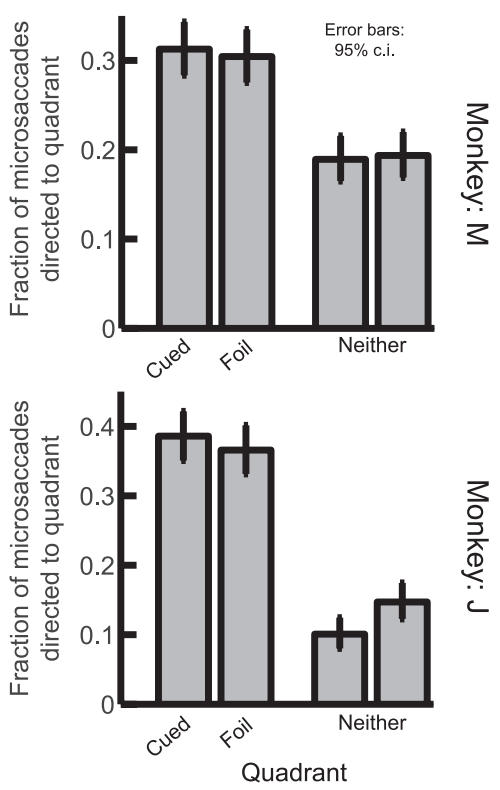

Figure 6. Even though microsaccades were significantly reduced in anticipation of motion pulse onset, the movements that did occur late in the trials reflected the behavioral relevance of spatial locations. Each panel shows that, for movements temporally proximal to motion pulse onset (i.e., late in the trials after all stimulus-induced transients have subsided), the likelihood of observing a microsaccade in a given direction was significantly increased if this direction corresponded to the locations where motion stimuli were expected (cued and foil). For neither cued nor foil directions, the likelihood of microsaccades was significantly less. Thus, when they did occur, microsaccades late in the trials reflected the behavioral relevance of the cued and foil locations compared with the other two locations. Error bars indicate $95 \%$ confidence intervals.

\section{Successful allocation of attention was reflected in} microsaccade directions

If microsaccades indeed reflect the sustained allocation of covert attention, then differences in microsaccade directions between the cued and foil locations late in the trials (when motion pulses occurred) should also correlate with the monkeys' success or failure in performing the task. To test this possibility, we repeated the analysis summarized in Figure 6 but sorted trials according to whether the monkey choices matched the cued or foil motion stimulus. When the monkeys successfully reported the direction of motion at the cued location, the fraction of microsaccades (around motion pulse onset) that were directed toward the cued location was significantly higher than the fraction of microsaccades directed toward the foil, and much higher than the fraction of movements toward the two distractor locations ("neither"). These differences were all significant (based on 95\% confidence intervals obtained from binomial distribution fits to the data). However, when the monkeys erroneously reported the direction of motion at the foil location, there were fewer microsaccades directed toward the cued location than toward the foil, but this difference only reached significance in monkey $\mathrm{M}$ (probably because monkey J had a strong inherent bias in microsaccade directions regardless of cueing) (see Materials and Methods). Thus, microsaccade directions at the end of the crucial delay period of the task appeared to reflect the monkeys' success in correctly allocating covert attention.

Microsaccades during the task were therefore associated with two, somewhat contradictory, behavioral effects: the general occurrence of microsaccades at motion pulse onset was associated with slightly impaired performance (Fig. 4), but the directions of microsaccades around pulse onset reflected the allocation of co-

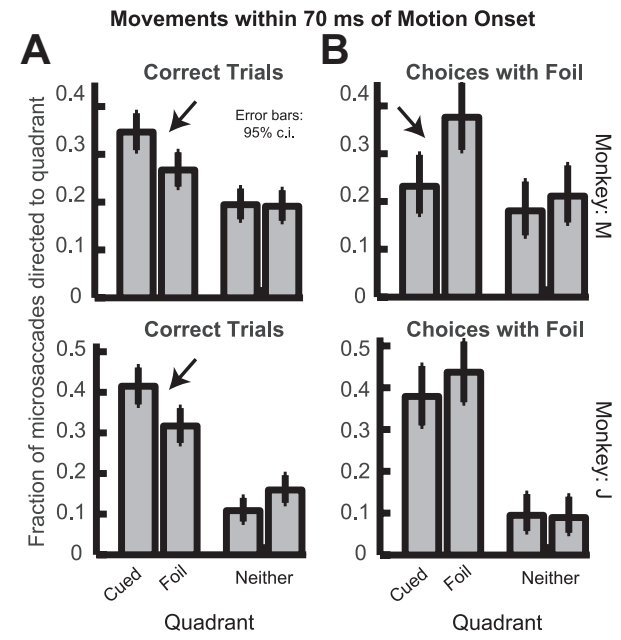

Figure 7. The directional modulation of late microsaccades observed in Figure 6 reflected the allocation of covert visual attention to the cued location. To test whether the directional biases in Figure 6 were related to covert attention, we repeated the same analyses but after dividing the trials based on correctness of response. On correct trials $(\boldsymbol{A})$, both monkeys showed significantly more microsaccades close to motion pulse onset that were directed toward the cued location than toward the foil location (arrows) or the remaining two irrelevant ones (Neither). When the monkeys erroneously reported the motion direction in the foil location $(\boldsymbol{B})$, there were more microsaccades close to motion pulse onset that were directed toward the foil location than toward the cued one, but this difference only reached significance (at the $95 \%$ level) in monkey M (arrow). Thus, success in the allocation of covert attention to the cued location was reflected in the distribution of microsaccades occurring late in the trials. Error bars indicate $95 \%$ confidence intervals.

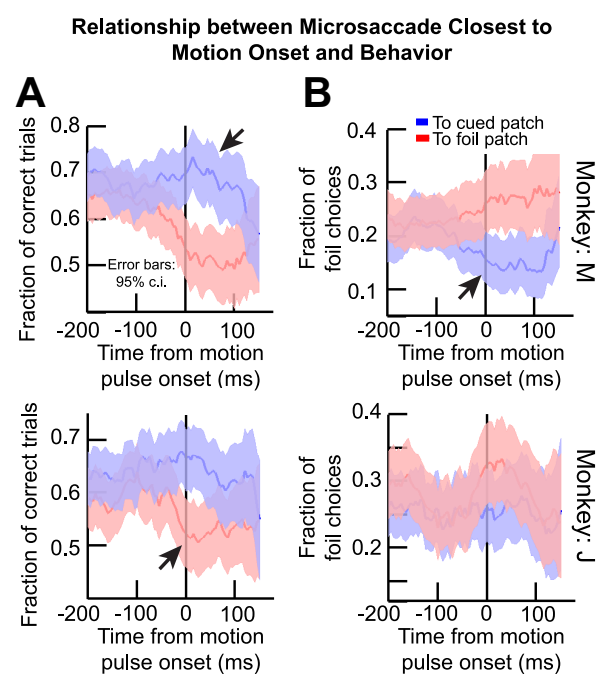

Figure 8. Asymmetric relation between microsaccade direction and behavioral performance. $A, A n$ analysis similar to that in Figure $4 B$ but after dividing trials based on whether a microsaccade around pulse onset was directed toward the cued or foil locations. Even though microsaccades around pulse onset were associated with a general decrease in performance (Fig. $4 B$ ), dividing the movements based on their direction revealed that this decrease did not occur for movements toward the cued location (perhaps due to more successful allocation of sustained attention, which was reflected in both the microsaccades and behavioral report) (blue). When microsaccades were directed toward the foil location, behavioral performance was suppressed when these movements occurred around motion pulse onset (red). $\boldsymbol{B}$, When plotting the fraction of foil choices instead of correct choices, the opposite pattern emerged in monkey M (but did not reach significance in monkey J). In other words, foil choices were more frequent when a microsaccade around motion pulse onset was directed toward the foil quadrant than when it was directed toward the cued location (reflecting a possible failure in allocating attention to the cued location and instead an erroneous allocation of attention to the foil). All conventions are otherwise similar to those in Figure $4 B$. 
vert attention (Figs. 6, 7) and predicted performance in the task. We next considered how these two effects might interact. Specifically, we tested whether microsaccades around motion pulse onset that were directed toward the cued location were associated with less reduction in success rate (as per Fig. $4 B$ ) than microsaccades directed toward the foil location. We repeated the analysis of Figure $4 B$ but for movements either in the direction of the cued location or in the direction of the foil location (Fig. 8A). On trials in which the microsaccades around motion pulse onset were in the direction of the cued location, success rate in both monkeys was higher than on trials in which the microsaccades around motion pulse onset were in the direction of the foil location (Fig. $8 \mathrm{~A}$, arrows). Moreover, in one of the monkeys $(\mathrm{M})$, the converse was also true: the fraction of choices with the motion direction at the foil location was higher when the microsaccades closest to motion pulse onset were directed toward the foil location than when they were directed toward the cued location (Fig. $8 B$, monkey $\mathrm{M})$. Thus, these results probably reflect that, on correctly performed trials, the successful allocation of attention influenced both the generation of microsaccades and the behavioral report. When the monkeys erroneously chose the foil motion stimuli, suggesting that attention may have been erroneously shifted to the foil location, microsaccades also tended to reflect that location. Moreover, these results also indicate that decreases in perceptual performance observed earlier in Figure 4 were mostly accounted for by microsaccades that were not directed toward the cued location; for microsaccades directed toward the cued location, the frequency of correct responses (Fig. 8) was not decreased as strongly as in Figure $4 B$.

\section{Microsaccades during the button press version of the covert attention task}

Microsaccades during the button press version of the task were mostly similar to those during the saccade version, but did exhibit some differences. One major similarity between the two tasks was the stereotypical pattern of microsaccades generated soon after stimulus onsets. This point is illustrated in Figure 9A, which presents the results of the same analysis as in Figure $3 A$, but applied to the button press data. The gray microsaccade rate curves in Figure $9 A$ are replicates of the curves in Figure $3 A$, included here to facilitate comparison between the two task variants. For early stimulus-induced changes in microsaccade rate, there were similar patterns to the saccade task. Specifically, cue onset, $0 \%$ motion patch onset, and cue offset all induced sets of microsaccades after latencies of $\sim 200-250 \mathrm{~ms}$. However, quantitatively, there were consistent differences between the two versions of the task. For example, cue and $0 \%$ motion patch onset in the button press version of the task induced more microsaccades than in the saccade task, and this effect was much stronger in monkey $\mathrm{M}$ than in
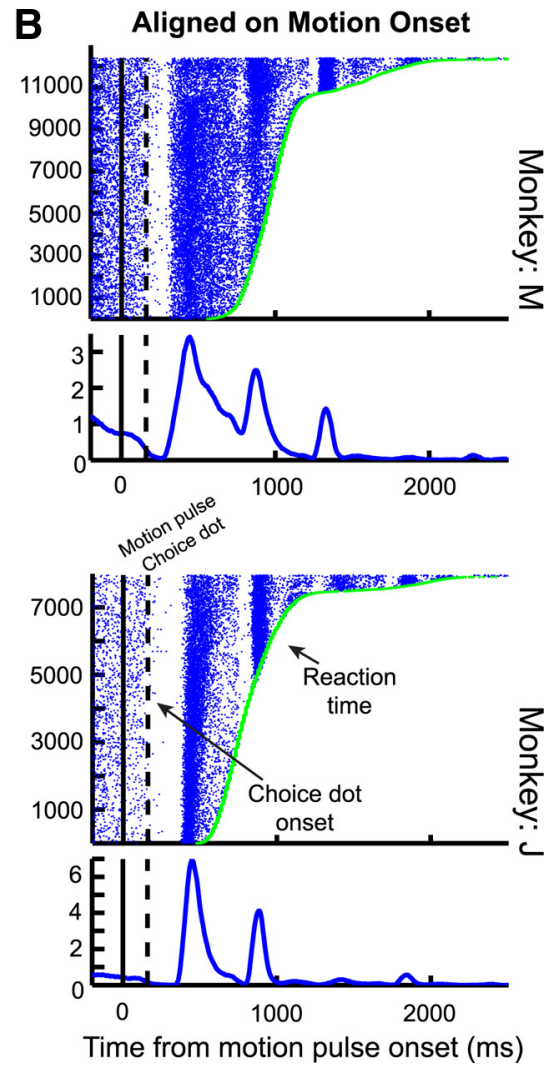

Time from motion pulse onset (ms)

Figure 9. Performing the same task in a different context (button press) modified the patterns of microsaccades that occurred.

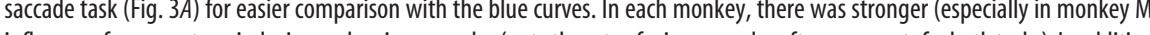
round the black dashed vertical line; arrows). $\boldsymbol{B}$, The same analyses of Figure $3 B$, but for the button press variant of the task. These analyses show that, after choice frame onset in this task, there were populations of movements evoked periodically until button press. These prebutton movements had very distinct directional characteristics (Fig. 10).

monkey J (who also showed strong stimulus-induced increases in microsaccades during these events in the saccade task). In addition, for both monkeys, the initial reduction in microsaccade rate shortly after cue offset (in anticipation of the shortest possible motion pulse time) was stronger in the button press version of the task.

The one major difference in microsaccades between the button press and saccade versions of the task was in the saccades occurring after motion pulse end. Comparison of the raster plots of Figure $9 A$ and Figure $3 A$ reveals that, in the button press task, there were populations of movements that were consistently generated after motion pulse end and before button press. These movements were time-locked to the motion pulse onset (or end), an effect that is much clearer when the data are aligned on trial end (Fig. 9B). As Figure 9B shows, after motion pulse end in the button press version, there were volleys of small eye movements that occurred periodically every few hundred milliseconds until the monkeys pressed a button. These movements were slightly larger than the saccades earlier in the trial (31.8 min arc vs 19.5 min arc in J; $17.7 \mathrm{~min}$ arc vs $12.7 \mathrm{~min}$ arc in $\mathrm{M} ; p<0.00001$ ), and therefore also larger than those in the saccade version of the task. Similar movements were not observed in the saccade version of the task, most likely because the shorter reaction times of saccades truncated this epoch of the trial.

The microsaccades that occurred after choice frame onset in the button press version of the task (Fig. $9 B$ ) consistently reflected 
A

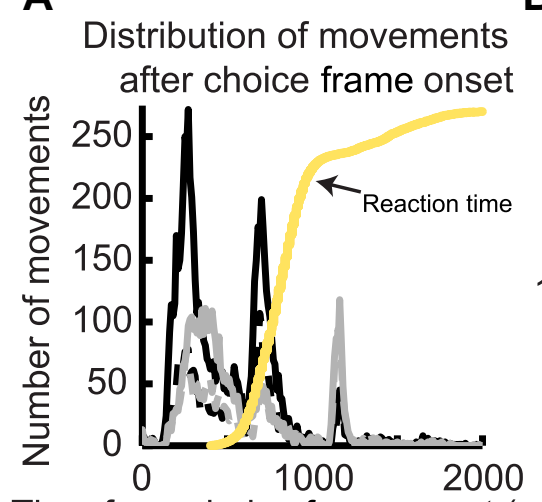

Time from choice frame onset (ms)

B

\section{Direction of first movement after choice frame onset}

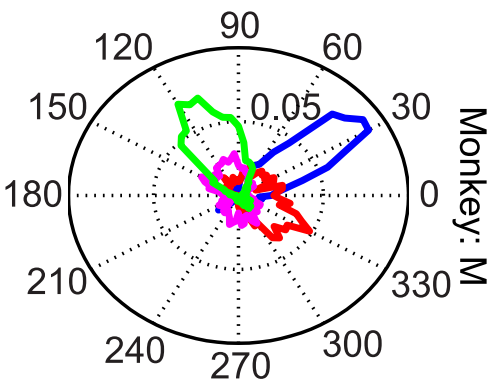

-Monkey chose quadrant 1 - Monkey chose quadrant 2 - Monkey chose quadrant 4

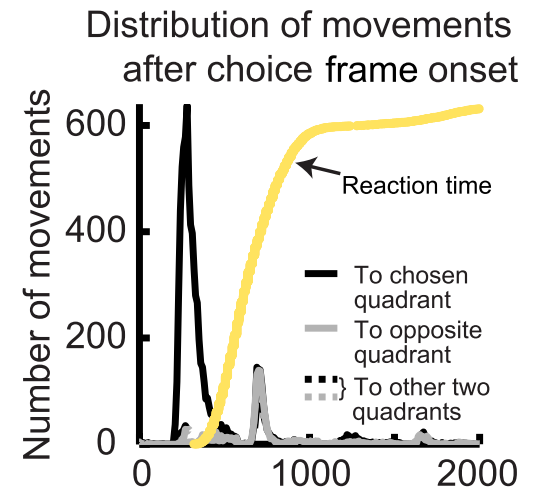

\section{Direction of first movement} after choice frame onset

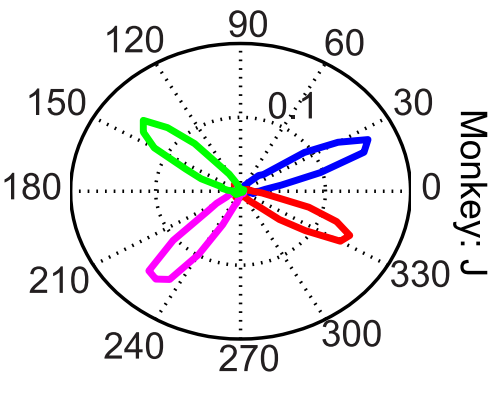

Time from choice frame onset $(\mathrm{ms})$

Figure 10. Microsaccade directions after choice frame onset in the button press variant of the task revealed a strong coordination between fixational eye movements and the chosen button to press. $A$, We plotted the distribution of post-choice-frame eye movements for each monkey as a function of time after choice frame onset ( $x$-axis) and movement direction (different curves as per the legend). In both monkeys, the first population of movements made after choice frame onset was predominantly directed toward the quadrant that was eventually chosen by the monkey with a button press. On longer RTs (RTs indicated by the yellow curve), the monkeys made subsequent volleys of movements that were either directed to the chosen or symmetrically opposite quadrant (probably reflecting refixation saccades). $\boldsymbol{B}$, To further illustrate the strong coupling between these post-choice-frame fixational eye movements and the quadrant chosen by button press, we plotted polar histograms of the angular direction of the first movement occurring after choice frame onset. Both monkeys showed a strong coupling between chosen quadrant and direction of first movement, except for movements into quadrant 4 in $\mathrm{M}$ (magenta), which were rare in this monkey.

the direction of the monkeys' choices. We examined the volleys of eye movements that occurred between motion pulse end and button press by analyzing the directions of these movements. We first plotted the histogram distributions of these movements as in Figure $9 B$ but after sorting the movements according to their direction (Fig. 10A). We found that the majority of microsaccades were congruent with the monkeys' selected button press direction, regardless of whether the choice was based on the cued or foil signals. For example, if the monkeys decided to press the button corresponding to quadrant 1 , microsaccades after choice frame onset were predominantly directed toward quadrant 1 . On longer reaction time trials, subsequent movements (after successive intervals of several hundred milliseconds) were either directed to the same quadrant again or to the diametrically opposite quadrant (which probably reflect refixation saccades) (Fig. 10A). Figure $10 B$ shows a polar histogram of the direction of the first movement to occur after motion pulse end, again dividing the movements based on which quadrant the monkey subsequently selected with a button press. In monkey J, there was an almost one-to-one correspondence between the direction of the first movement after motion pulse end and the chosen button press quadrant. In monkey $\mathrm{M}$, a similar relationship was observed except that this monkey had a tendency not to generate microsaccades toward quadrant 3 , and instead exhibited a bias for upward movements (Fig. 10B). Thus, when the monkeys were instructed to maintain fixation while preparing their button press responses, the microsaccades that occurred before such responses almost exclusively signaled the impending choice.

Other aspects of microsaccade direction relative to stimulus onsets and behavioral relevance of the spatial locations were very similar in the button press and saccade variants of the task. For example, Figure 11 plots the time course of microsaccade directions after cue onset in the button press variant of the task, using the same conventions as in Figure 5; the dashed colored curves show the results from the saccade version of the task to facilitate comparisons between the two task versions. The directions of microsaccades were again modulated by the cues and the behavioral relevance of the cued and foil locations, and these effects were at least as strong in the button press version of the task as in the saccade version, for both monkeys. Thus, behavioral context did not dramatically alter the modulations of microsaccade direction that we observed in the two versions of the same task.

\section{Discussion}

We investigated the occurrence of microsaccades during a demanding covert attention task requiring prolonged fixation (Lovejoy and Krauzlis, 2010) and found that microsaccades occurred in very orderly patterns related to stimulus events in this task. Moreover, microsaccade generation was influenced by task requirements, as indicated by the marked reduction in microsaccades as the monkey anticipated the probe stimulus, and the biases in microsaccade direction toward behaviorally relevant locations. The reduction in microsaccade rate is reminiscent of studies demonstrating voluntary suppression of microsaccades in humans (Steinman et al., 1967) and may have occurred in our experiments to minimize possible effects of microsaccadic suppression (Zuber and Stark, 1966; Beeler, 1967; Hafed and Krauzlis, 2010). As we discuss below, these results clarify the role of cognition in influencing microsaccades, how and why these movements may be generated under changing stimulus and cognitive states, and the functional role of these movements in visual performance. These results also suggest that the analysis of microsaccades in visual neuroscience experiments may often be necessary for the proper interpretation of experimental results.

\section{The influence of cognition on microsaccades}

Our analyses revealed several influences on microsaccade generation related to the behavioral relevance of stimuli and locations. Some of these influences appeared to be bottom-up or stimulus driven. For example, we observed consistent sets of "stimulus- 


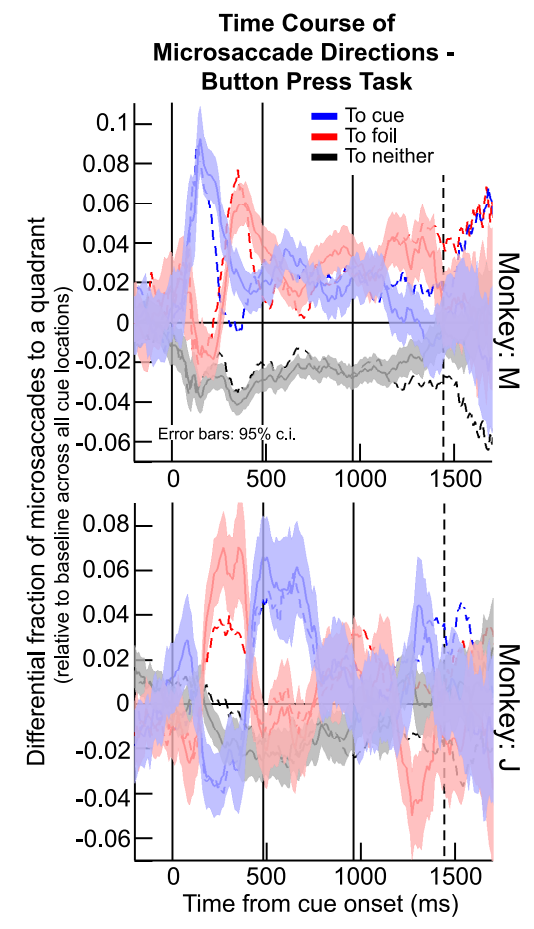

Figure 11. Microsaccade directions after cue onset reflected the cued and foil locations in the button press variant of the task, just as in the saccade variant. For each monkey, we repeated the same analysis of Figure 5 but for the button press case. For comparison, the colored dashed curves show the data from Figure 5 for the saccade version of the task. Microsaccades in both tasks showed temporal evolutions that were very similar. Figure conventions are the same as those in Figure 5.

induced" microsaccades that occurred shortly after cue onset, random dot patch onset, and cue offset (Figs. 3, 9). The generation of these microsaccades appeared to be partly reflexive, because they repeatedly occurred on thousands of trials containing the same stimulus events. However, the modulations in their directions toward the cued location suggest that they may also be influenced by voluntary or top-down influences (Figs. 5, 11). Similar influences have been found in humans (Hafed and Clark, 2002; Engbert and Kliegl, 2003) and could arise as a result of the effects of attention on neural activity in sensory-motor structures. For example, stimulus-induced visual bursts in the SC are modulated by attention (Fecteau et al., 2004; Fecteau and Munoz, 2005; Boehnke and Munoz, 2008). We hypothesize that the modulation of these visual bursts with attention introduces a bias in the SC representation of the fixated target away from "straight ahead," which is sufficient to trigger very small saccades (Hafed and Krauzlis, 2008; Hafed et al., 2008, 2009). Thus, early modulations in microsaccade direction in our task related to behaviorally relevant locations may reflect the influence of covert attention on stimulus-induced visual activity in sensory-motor structures, such as the SC.

We also observed significant top-down influences of behaviorally relevant locations on microsaccades. Specifically, during the delay period of our task, long after the stimulus-induced modulations in microsaccade rate had subsided, the directions of microsaccades were biased toward the expected locations of the motion pulses (Figs. 6-8). Moreover, the successful allocation of attention to the cued location, assayed by calculating the percentage of correctly performed trials, was reflected in the directions of these microsaccades: when microsaccades were directed toward the cued location close to motion pulse onset, the monkeys were more likely to succeed in discriminating the cued signal, whereas when the movements were directed away from the cued location (for example, to refixate the fixation spot after a movement away from it), the monkeys were less likely to succeed. These results constitute a direct correlate of human results in which a relationship between microsaccade direction and task performance was explored (Hafed and Clark, 2002), and they lend support to the conclusion that microsaccades are influenced by the sustained, top-down allocation of covert attention. This influence of sustained top-down or voluntary attention on microsaccades is probably also mediated by sensory-motor structures. For example, in the SC, sustained covert attention can elevate baseline firing rates at the attended locations (Kustov and Robinson, 1996; Ignashchenkova et al., 2004), which could affect the overall balance of activity in representing the fixated target location around the rostral SC (Hafed and Krauzlis, 2008; Hafed et al., 2008, 2009). Thus, our results establish that the top-down allocation of attention influences microsaccade generation in the monkey, as well as in humans (Hafed and Clark, 2002).

Our task design, which involved a perceptual discrimination stimulus presented very briefly any time between $\sim 1440$ and $\sim 4000$ ms after stimulus onset, revealed another aspect of potential voluntary control of microsaccades in monkeys. Specifically, we observed a dramatic drop of microsaccade rate during the interval in which the brief motion pulse was expected (Figs. 3, 9). The difficulty in correctly discriminating such a brief stimulus, combined with the possible effect of microsaccades on task performance (Figs. 4, 8), suggests that this reduction in microsaccade rate may have involved voluntary suppression of these movements. Voluntary suppression of microsaccades in humans has been described previously (Steinman et al., 1967; Pastukhov and Braun, 2010), but it remains unclear to what extent such voluntary control over microsaccades exists, and the detailed physiological mechanisms responsible are unknown. For example, is it possible to voluntarily generate a movement of the same size scale as microsaccades (Haddad and Steinman, 1973)? If this is the case [as recent evidence in humans suggests (Ko et al., 2010)], then this may also explain some of the individual differences between monkeys that we observed in our experiments. For example, even though early stimulus-induced microsaccades were consistently biased toward the cued and foil locations in both monkeys (Figs. 5, 11), the temporal ordering of these biases differed between them such that the initial bias was first toward the cue and then the foil in one monkey and the opposite in the other. If microsaccades are under voluntary control, then this difference may reflect the different strategies used by the two monkeys during the early epochs in the trial, when no perceptual discrimination was expected to occur.

Regardless of whether individual microsaccades are under voluntary control, the observation of reduced microsaccade rates in later trial epochs in our tasks suggests that the role of microsaccades in vision may extend beyond the popular retinal image refreshing hypothesis (Martinez-Conde et al., 2004, 2006; Engbert and Mergenthaler, 2006). In fact, under retinal image stabilization conditions, which cause perceptual fading, microsaccade frequency declines rather than increases (Poletti and Rucci, 2010). Instead, microsaccades may be necessary for discriminating fine visual detail (Rucci et al., 2007; Ko et al., 2010). Thus, these movements may be used by the visual and oculomotor systems as part of a more sophisticated fixation strategy: one that attempts to balance different competing constraints such as the maintenance of spatial accuracy of gaze position, the prevention of peripheral image fading, the enhancement of perceptual sta- 
bility in the face of constant eye movement, and the avoidance of the detrimental effects of phenomena such as microsaccadic suppression on perceptual performance.

\section{Behavioral context and its influence on microsaccades}

In the button press version of our task, microsaccades exhibited another modulation, characterized by a strong coupling between these eye movements and the manual response. Specifically, the directions of microsaccades generated while the monkeys were preparing their button presses were highly correlated with the location of the chosen button. This directional effect was the strongest directional modulation for microsaccades we found across all analyses, and a likely reason for the strength of this effect is the onset of a salient fixation stimulus during button press preparation (see Materials and Methods). The foveal visual stimulus had clear landmarks (corners of a square) that may have provided visual features to guide eye movements as the monkeys prepared their button press response. However, even given this influence of visual guidance, the observation of directional coupling with the pressed button suggests that the monkeys preferred to generate small eye movements that were associated with the manual response, despite the large spatial dissociation between the two response modalities. This phenomenon, which may have been a consequence of the fact that the monkeys were highly trained on the saccade version of the task first, warrants further investigation, especially given that many studies of microsaccades and their role in vision require button presses (for example, to indicate a percept or lack thereof).

\section{Controlling for microsaccades in experiments}

Finally, our results support the view that fixation is an active process, which may well be under strong voluntary and cognitive control. This viewpoint has important consequences for the design and interpretation of visual neuroscience experiments. For example, in studies of neuronal coding in sensory areas, correlated activity among populations of neurons, and how it may be modulated by cognitive processes (Cohen and Maunsell, 2009; Mitchell et al., 2009), plays an important role in perception and subsequent action. Because microsaccades move the entire retinal image every time they occur, these movements effectively introduce strong periodic correlations in sensory activity. While this means that fixational eye movements can be a nuisance in visual neuroscience experiments, it could also be the case that such large correlations in neural activity caused by microsaccades are a property that is actively controlled, or exploited, by the visual and oculomotor systems.

\section{References}

Barlow HB (1952) Eye movements during fixation. J Physiol 116:290-306. Beeler GW Jr (1967) Visual threshold changes resulting from spontaneous saccadic eye movements. Vision Res 7:769-775.

Boehnke SE, Munoz DP (2008) On the importance of the transient visual response in the superior colliculus. Curr Opin Neurobiol 18:544-551.

Bosman CA, Womelsdorf T, Desimone R, Fries P (2009) A microsaccadic rhythm modulates gamma-band synchronization and behavior. J Neurosci 29:9471-9480.

Brien DC, Corneil BD, Fecteau JH, Bell AH, Munoz DP (2009) The behavioral and neurophysiological modulation of microsaccades in monkeys. J Eye Mov Res 3:1-12.

Cohen MR, Maunsell JH (2009) Attention improves performance primarily by reducing interneuronal correlations. Nat Neurosci 12:1594-1600.

Corneil BD, Munoz DP, Chapman BB, Admans T, Cushing SL (2008) Neuromuscular consequences of reflexive covert orienting. Nat Neurosci 11:13-15.
Cui J, Wilke M, Logothetis NK, Leopold DA, Liang H (2009) Visibility states modulate microsaccade rate and direction. Vision Res 49:228-236.

Deaner RO, Platt ML (2003) Reflexive social attention in monkeys and humans. Curr Biol 13:1609-1613.

Diamond MR, Ross J, Morrone MC (2000) Extraretinal control of saccadic suppression. J Neurosci 20:3449-3455.

Dimigen O, Valsecchi M, Sommer W, Kliegl R (2009) Human microsaccaderelated visual brain responses. J Neurosci 29:12321-12331.

Ditchburn RW, Ginsborg BL (1953) Involuntary eye movements during fixation. J Physiol 119:1-17.

Efron B, Tibshirani RJ (1993) An introduction to the bootstrap. New York: Chapman and Hall.

Engbert R, Kliegl R (2003) Microsaccades uncover the orientation of covert attention. Vision Res 43:1035-1045.

Engbert R, Mergenthaler K (2006) Microsaccades are triggered by low retinal image slip. Proc Natl Acad Sci U S A 103:7192-7197.

Fecteau JH, Munoz DP (2005) Correlates of capture of attention and inhibition of return across stages of visual processing. J Cogn Neurosci 17:1714-1727.

Fecteau JH, Bell AH, Munoz DP (2004) Neural correlates of the automatic and goal-driven biases in orienting spatial attention. J Neurophysiol 92:1728-1737.

Haddad GM, Steinman RM (1973) The smallest voluntary saccade: implications for fixation. Vision Res 13:1075-1086.

Hafed ZM (2011) Mechanisms for generating and compensating for the smallest possible saccades. Eur J Neurosci 33:2101-2113.

Hafed ZM, Clark JJ (2002) Microsaccades as an overt measure of covert attention shifts. Vision Res 42:2533-2545.

Hafed ZM, Krauzlis RJ (2008) Goal representations dominate superior colliculus activity during extrafoveal tracking. J Neurosci 28:9426-9439.

Hafed ZM, Krauzlis RJ (2010) Microsaccadic suppression of visual bursts in the primate superior colliculus. J Neurosci 30:9542-9547.

Hafed ZM, Goffart L, Krauzlis RJ (2008) Superior colliculus inactivation causes stable offsets in eye position during tracking. J Neurosci 28:8124-8137.

Hafed ZM, Goffart L, Krauzlis RJ (2009) A neural mechanism for microsaccade generation in the primate superior colliculus. Science 323:940-943.

Herrington TM, Masse NY, Hachmeh KJ, Smith JE, Assad JA, Cook EP (2009) The effect of microsaccades on the correlation between neural activity and behavior in middle temporal, ventral intraparietal, and lateral intraparietal areas. J Neurosci 29:5793-5805.

Horowitz TS, Fine EM, Fencsik DE, Yurgenson S, Wolfe JM (2007) Fixational eye movements are not an index of covert attention. Psychol Sci 18:356-363.

Ignashchenkova A, Dicke PW, Haarmeier T, Thier P (2004) Neuronspecific contribution of the superior colliculus to overt and covert shifts of attention. Nat Neurosci 7:56-64.

Kagan I, Gur M, Snodderly DM (2008) Saccades and drifts differentially modulate neuronal activity in V1: effects of retinal image motion, position, and extraretinal influences. J Vis 8:19.1-25.

Ko HK, Poletti M, Rucci M (2010) Microsaccades precisely relocate gaze in a high visual acuity task. Nat Neurosci 13:1549-1553.

Krauzlis RJ (2003) Neuronal activity in the rostral superior colliculus related to the initiation of pursuit and saccadic eye movements. J Neurosci 23:4333-4344.

Krauzlis RJ, Miles FA (1996) Release of fixation for pursuit and saccades in humans: evidence for shared inputs acting on different neural substrates. J Neurophysiol 76:2822-2833.

Kustov AA, Robinson DL (1996) Shared neural control of attentional shifts and eye movements. Nature 384:74-77.

Laubrock J, Engbert R, Kliegl R (2005) Microsaccade dynamics during covert attention. Vision Res 45:721-730.

Laubrock J, Kliegl R, Rolfs M, Engbert R (2010) When do microsaccades follow spatial attention? Atten Percept Psychophys 72:683-694.

Leopold DA, Logothetis NK (1998) Microsaccades differentially modulate neural activity in the striate and extrastriate visual cortex. Exp Brain Res 123:341-345.

Lovejoy LP, Krauzlis RJ (2010) Inactivation of primate superior colliculus impairs covert selection of signals for perceptual judgments. Nat Neurosci 13:261-266. 
Martinez-Conde S, Macknik SL, Hubel DH (2000) Microsaccadic eye movements and firing of single cells in the striate cortex of macaque monkeys. Nat Neurosci 3:251-258.

Martinez-Conde S, Macknik SL, Hubel DH (2004) The role of fixational eye movements in visual perception. Nat Rev Neurosci 5:229-240.

Martinez-Conde S, Macknik SL, Troncoso XG, Dyar TA (2006) Microsaccades counteract visual fading during fixation. Neuron 49:297305.

Mitchell JF, Sundberg KA, Reynolds JH (2009) Spatial attention decorrelates intrinsic activity fluctuations in macaque area V4. Neuron 63:879-888.

Pastukhov A, Braun J (2010) Rare but precious: microsaccades are highly informative about attentional allocation. Vision Res 50:1173-1184.

Poletti M, Rucci M (2010) Eye movements under various conditions of image fading. J Vis 10:6 1-18.

Ratliff F, Riggs LA (1950) Involuntary motions of the eye during monocular fixation. J Exp Psychol 40:687-701.
Ross J, Morrone MC, Goldberg ME, Burr DC (2001) Changes in visual perception at the time of saccades. Trends Neurosci 24:113-121.

Rucci M, Iovin R, Poletti M, Santini F (2007) Miniature eye movements enhance fine spatial detail. Nature 447:851-854.

Steinman RM, Cunitz RJ, Timberlake GT, Herman M (1967) Voluntary control of microsaccades during maintained monocular fixation. Science 155:1577-1579.

Tse PU, Sheinberg DL, Logothetis NK (2002) Fixational eye movements are not affected by abrupt onsets that capture attention. Vision Res 42:1663-1669.

Van Gisbergen JA, Robinson DA, Gielen S (1981) A quantitative analysis of generation of saccadic eye movements by burst neurons. J Neurophysiol 45:417-442.

Zuber BL, Stark L (1965) Microsaccades and the velocity-amplitude relationship for saccadic eye movements. Science 150:1459-1460.

Zuber BL, Stark L (1966) Saccadic suppression: elevation of visual threshold associated with saccadic eye movements. Exp Neurol 16:65-79. 